\title{
Metastatic basal cell carcinoma: report of a case responding to chemotherapy
}

\author{
R. L. Woods \\ M.B., M.R.C.P.
}

\author{
J. F. Stewart \\ M.B. B.S.
}

\author{
Ludwig Institute for Cancer Research, \\ Blackburn Building, University of Sydney, NSW 2006
}

\begin{abstract}
Summary
Metastases of basal cell carcinoma are very uncommon. Only 10 reported cases have been treated with cytotoxic chemotherapy, and no good responses have been documented. The case is presented of a 54-year-old male who had several basal cell carcinomas (BCCs) removed surgically and who, 12 years after his first presentation, developed respiratory symptoms related to metastatic pulmonary BCCs. These failed to respond to initial chemotherapy with methotrexate, bleomycin, and vincristine, but regressed when treated with cyclophosphamide and cis-diamine dichloroplatinum.
\end{abstract}

\section{Introduction}

Basal cell carcinomas are the commonest skin malignancy in Caucasians, accounting for approximately $70 \%$ of skin cancers. The natural history of basal cell carcinoma is one of local invasion and recurrence, and metastases are rare. Only 109 cases of documented metastatic basal cell carcinoma have been reported (Safai, 1977) and, of these, the majority metastasize to lymph nodes. Only 34 cases have been reported involving the lung or pleura. Ten of these cases have been treated with cytotoxic chemotherapy, but none has responded to this modality. A case is now reported of the first documented response of metastatic basal cell carcinoma to cytotoxic chemotherapy.

\section{Case report}

A 54-year-old Caucasian presented in 1966 with a skin lesion at the angle of his mouth which was removed and histologically diagnosed as a basal cell carcinoma. The lesion recurred locally and, over the next 5 years, 9 further surgical excisions were performed in an attempt to remove recurrent lesions.

In June 1975, he re-presented with a 12-month history of right sub-mandibular swelling. A firm mass measuring $2 \mathrm{~cm}$ in diameter which was fixed to the lower border of the mandible was palpable, and biopsy showed invasive basal cell carcinoma. No cutaneous lesions were present at this time. This was removed with a partial mandibulectomy and $\overrightarrow{\vec{\omega}}$ rotational neck flap and histological examination confirmed that tumour invasion on the mandible $\frac{O}{3}$ had occurred. He remained well until January 1978, ㄱ. when he gave a history of one month's right pleuritic 9 chest pain with a cough productive of green sputum of and weight loss of $13 \mathrm{~kg}$ over the previous 3 months. Clinical examination suggested consolidation in the $N$ mid zone of the right lung and this was confirmed o on a chest X-ray which also showed several small rounded opacities ranging in diameter from $0.5 \vec{D}$ to $2 \mathrm{~cm}$ which were compatible with metastases. 을 Sputum examination showed coagulase-positive $\overrightarrow{\vec{\theta}}$

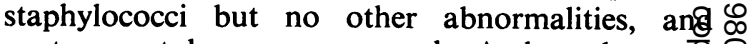
sputum cytology was normal. A bronchosco revealed a narrowed lateral basal bronchus in the right lower lobe and biopsy showed basal cen carcinoma. In view of his systemic symptoms, chemotherapy was commenced with methotrexate, vincristine and bleomycin. Following 3 courses of $\propto$ these drugs, there was slight progression in the size of $\overrightarrow{\vec{a}}$ the pulmonary lesions, and chemotherapy was $\frac{3}{3}$ changed to cyclophosphamide $500 \mathrm{mg} / \mathrm{m}^{2}$ i.v. and cis-diamine-dichloroplatinum (cis-DDP) 100 후 $\mathrm{mg} / \mathrm{m}^{2}$ i.v. both on day 1 , and repeated after $21 \%$ days. A partial response $(>50 \%$ reduction in the 3 . sum of the products of the maximum perpendicular tumour diameters) of all pulmonary metastases 3 . occurred after 2 courses of this and he became $\delta$ asymptomatic and started to gain weight. Two further courses of cyclophosphamide and cis-DDP 음 were given. He remains well 12 months later with no symptoms. Opacities are still present on chest $\mathrm{X}$-ray but are markedly reduced in size from the time of his presentation.

\section{Discussion}

This case report illustrates some of the difficulties 0 of managing patients with metastatic basal cell 0 carcinomas. The problem is usually one of local $\frac{\vec{D}}{\mathbb{D}}$ recurrence and invasion and the treatment of this $\stackrel{?}{+}$ is with local excision or radiotherapy. If adequate $\square$ control of the initial lesion is obtained the outlook is excellent, but these lesions can prove resistant to 
therapy and local recurrence may eventually be fatal. Development of metastatic basal cell carcinomas is associated with a poor prognosis with a median survival time of approximately 10 months (Costanza et al., 1974). Systemic chemotherapy has not been commonly used in basal cell carcinomas although topical cytotoxics have been used with good results for non-metastasizing lesions, and 5-fluorouracil has been particularly valuable used in this way (Klein, 1968). In view of the poor prognosis in the present case it was felt that systemic chemotherapy was justified in spite of the poor results reported to date. Ten reported cases have been treated with cytotoxic chemotherapy and the drugs most commonly used have been bleomycin, methotrexate and 5fluorouracil. None of these 10 cases has responded to the chemotherapy and thus the choice of drugs in this case was purely speculative. The authors had previously noted that, in some patients, concomitant basal cell carcinomas had sometimes regressed during treatment for squamous cell carcinoma of the head and neck. The schedule which the authors had been using for head and neck cancers at that stage was the one previously described with methotrexate, bleomycin, and vincristine and this was therefore used as initial chemotherapy, but produced no benefit. These lesions are known to be sensitive to radio-therapy and it might therefore be thought that the so-called radiomimetic drugs might be effective. Only 2 cases have been reported in which alkylating agents have been used (Costanza et al., 1974; Assor, 1967). No response occurred in the first case which was a lesion involving bone treated with mechlorethamine hydrochloride. The second patient had pulmonary metastases which were initially treated with 5-fluorouracil followed by bleomycin. Cyclophosphamide and methotrexate were only used preterminally without success. The present authors therefore felt justified in using an alkylating agent, and cyclophosphamide was administered; they also gave cis-platinum, which is considered by some to be a bifunctional alkylating agent. The decrease in the size of the lesions which was observed and the improvement in the patient's symptoms confirm that these drugs have been effective.

\section{References}

AssoR, D. (1967) Basal cell carcinoma with metastasis to bone-Report of two cases. Cancer, 20, 2125.

Costanza, M.E., Dayal, Y., Binder, S., Nathanson, L., SAFAI, B., \& Good, R.A. (1974) Metastatic basal cell carcinoma: Review, report of a case and chemotherapy. Cancer, 34, 230.

Klein, E. (1968) Tumors of the skin-IX. Local cytostatic therapy of cutaneous and normal post-malignant and malignant lesions. New York State Journal of Medicine, 68, 886.

SAFAI, B. (1977) Basal cell carcinoma with metastasisReview of literature. Archives of Pathology and Laboratory Medicine, 101, 327. 